\title{
An Evaluation of Plaque Removal Efficacy of Five Commercially Available Toothbrushes: A Comparative Clinical Study
}

\author{
Swarna Chakrapani ${ }^{1, *}$, Tejaswin Polepalle ${ }^{2}$, Lakshmikanth Kolaparthy ${ }^{2}$, RupaSruthi Kuntcham ${ }^{1}$, \\ Chaitanya Adurty ${ }^{1}$, Sindhuri Sirigadha ${ }^{1}$ \\ ${ }^{1}$ Department Of Periodontology, SIBAR Institute of Dental Sciences, Takkellapadu, Guntur, Andhra Pradesh, India \\ ${ }^{2}$ Department of Periodontology, Sibar Dental College \& Hospital, Guntur, Andhra Pradesh, India \\ *Corresponding author: chakrapani2020@gmail.com
}

Received October 10, 2014; Revised October 25, 2014; Accepted October 28, 2014

\begin{abstract}
Background and Objectives - The aim of the study is to compare the plaque removal efficacy of five commercially available tooth brushes. Materials \& Methods -The study group consisted of 75 subjects with age ranging 17 to 27 years distributed into five groups- Group A [Colgate Supershine], Group B [Colgate Zig zag], Group C [Oral B Allrounder], Group D [Oral B Fresh Clean], Group E [Pepsodent double action]. Study was single blinded, randomized clinical trial. Turesky- Gilmore- Glickmann Modification of Quigley-Hein Plaque Index and Modified Sulcular bleeding index were assessed at baseline, 4 weeks and 8 weeks. Results - All brushes showed significant reduction in plaque score over 8 weeks. The percentage of plaque reduction from baseline to 4 weeks for Group A, B, C, D \& E is 58.74, 61.95, 57.86, 50.28 \& 53.59 respectively. The percentage of plaque reduction from baseline to 8 weeks for Group A, B, C, D \& E is 67.83, 76.11, 62.86, 56.42 \& 60.13 respectively. The percentage of plaque reduction from 4 weeks to 8 weeks for Group A, B, C, D \& E is 22.03, 37.21, 11.86, 12.36 \& 14.08 respectively. However, the reduction of plaque scores is greater in Group B [76\%] when compared to other groups. Conclusion - Within the limitations of the study, the data derived from the study shows that all the tooth brushes has shown decrease in plaque scores. In comparison, Colgate Zig zag [Group B] has some beneficial effects in reduction of plaque scores.
\end{abstract}

Keywords: toothbrush, plaque, bristle design

Cite This Article: Swarna Chakrapani, Tejaswin Polepalle, Lakshmikanth Kolaparthy, RupaSruthi Kuntcham, Chaitanya Adurty, and Sindhuri Sirigadha, "An Evaluation of Plaque Removal Efficacy of Five Commercially Available Toothbrushes: A Comparative Clinical Study.” International Journal of Dental Sciences and Research, vol. 2, no. 6A (2014): 15-20. doi: 10.12691/ijdsr-2-6A-4.

\section{Introduction}

Dental plaque is considered as a prime etiological factor in establishment of gingivitis which may eventually lead to periodontitis. [1] The bacterial plaque initiates an inflammatory process in the supporting structures of the tooth and if allowed to continue, ultimately may lead to loss of teeth. Hence, the health of periodontal tissues depends on effective plaque removal. Of all the mechanical methods, plaque removal using mechanical tooth brushes stands to be primary beneficial home care method.

The bristle toothbrushes appeared about the year 1600 in china. It was patented in America in 1857. Fredick Wilhelm Tornberg was credited for designing the first mechanical tooth brush in 1885. [2] Tooth brushes were evolved enormously in its shape, size and design since its launch. They have undergone great degree of sophistication over a period of time. Various designs in head of the toothbrush like two-headed, triple-headed along with differences in bristle patterns like V-shaped, Multitufted, Two-level, curved, Circular \& Diamond, Trimmed had made their appearance in the history of toothbrush evolution. However, the pioneers of today's toothbrushes were developed in the 1930s.

Patients without any professional advice usually choose brushes depending on its cost, availability, habit, and advertising claims. However, varying bristle patterns made different types of brushes flooded into the market claiming for better plaque removal efficacy. This may leave common man in quandary in selecting a toothbrush for which usually they may seek a professional advice. With the constant development in new designs, the knowledge of dental professionals should be upgraded for providing a better solution for the patients.

Various studies were conducted in testing the plaque removal efficacy of manual toothbrushes. Nevertheless, the results seem to be conflicting. There is, however, still insufficient evidence that one specific tooth brush design is superior to another. The use of toothbrush along with appropriate brushing technique ensures better plaque removal. The present study aims to evaluate the plaque 
removal efficacy of five commercially available tooth brushes.

\section{Materials \& Methods}

The study was a randomized 5 cell, examiner blind clinical trial and self-funded. Seventy five dental students consisting of 15 males and 60 females, randomly distributed into 5 groups with age ranging from 17 to 27 years were included in the study. Distribution was done randomly using lottery method. The study protocol was approved by institutional ethical committee. Participants were explained about the study and later informed and written concerns were taken. All the participants were dentate with minimum 28 numbers of teeth. Participants with 1) Previous usage of antibiotics and antiinflammatory drugs 6 months before to eliminate the their effect on plaque formation 2) any systemic conditions 3) Periodontal diseases 4) with severe crowding 5) Patients undergoing orthodontic treatment 6) Any adverse habits 7) Other supplemental plaque control aids were excluded from the study.

Five commercially available toothbrushes were included in the study. They are

1. Colgate Supershine ${ }^{*}$ - Single circular bristles GROUP A

2. Colgate Zig zag ${ }^{\dagger}-\mathrm{V}$ rim bristles - GROUP B

3. Oral B Allrounder ${ }^{\ddagger}$ - double circular bristles GROUP C

4. Oral B Fresh Clean ${ }^{\ddagger}-$ Flat Rim bristles - GROUP D

5. Pepsodent Double Action ${ }^{\S}$ - Double Circular Bristles. - GROUP E

To achieve standardized conditions, each participant was given a common dentifrice [Colgate Dental cream ${ }^{\dagger}$ ]. Participants were allotted tooth brushes by a nonparticipating dentist. Modified bass technique was demonstrated and instructed to follow the same for 3 minutes. Turesky- Gilmore- Glickmann Modification of Quigley-Hein Plaque Index[PI] [3] and Modified Sulcular bleeding index[BI] [4] were assessed at baseline, 4 weeks \& 8 weeks.

\section{Statistical Analysis}

The plaque removal was tested for statistical significance using non parametric Wilcoxon rank test for paired samples. The differences in plaque reduction between the groups were tested for statistical significance by non-parametric Kruskal wallis $\mathrm{H}$ test with significance level of $p \leq 0.05$.

\section{Results}

All seventy five participants completed the study protocol. Age of the participants ranged from 17 to 21 and the mean age is $18.32 \pm 0.95$ [SD]. The mean plaque index at baseline for Group A, B, C, D and E are 1.43, 1.13, 1.4, 1.79 and 1.53 respectively. The mean plaque index at four weeks for Group A, B, C, D and E are 0.59, 0.43, 0.59,

\footnotetext{
${ }^{*}$ Colgate Palmolive [India] Ltd.

${ }^{\dagger}$ A Rialto Enterprises Pvt. Ltd

$\S$ Hindustan Unilever Ltd.
}

0.89 and 0.71 respectively. The mean plaque index at eight weeks for Group A, B, C, D and E are 0.46, 0.27, $0.52,0.78$ and 0.61 respectively.

The percentage of plaque reduction from baseline to four weeks for Group A, B, C, D and E is 58.74, 61.95, 57.86, 50.28 and 53.59 respectively. The percentage of plaque reduction from baseline to eight weeks for Group A, B, C, D \& E is 67.83, 76.11, 62.86, 56.42 and 60.13 respectively. The percentage of plaque reduction from four weeks to eight weeks for Group A, B, C, D and E is 22.03, 37.21, 11.86, 12.36 and 14.08 respectively. The reduction in all the groups from baseline to four weeks, four weeks to eight weeks and from baseline to eight weeks is statistically significant.

The mean bleeding index at baseline for Group A, B, C, $\mathrm{D}$ and $\mathrm{E}$ are $0.48,0.37,0.23,0.33$ and 0.22 respectively. The mean bleeding index at four weeks for Group A, B, C, $\mathrm{D}$ and $\mathrm{E}$ are $0.34,0.2,0.18,0.22$ and 0.17 respectively. The mean bleeding index at eight weeks for Group A, B, C, D and $\mathrm{E}$ are $0.26,0.16,0.16,0.18$ and 0.12 respectively. The reduction in all the groups from baseline to four weeks, four weeks to eight weeks and from baseline to eight weeks is statistically significant.

The percentage of reduction of bleeding from baseline to four weeks for Group A, B, C, D \& E is 29.17, 35.14, $21.74,33.33$ and 22.73 respectively. The percentage of reduction of bleeding from baseline to eight weeks for Group A, B, C, D and E is 45.83, 51.53, 30.43, 45.46 and 45.45 respectively. The percentage of reduction of bleeding from four weeks to eight weeks for Group A, B, $\mathrm{C}, \mathrm{D}$ and $\mathrm{E}$ is 23.53, 30, 11.11, 18.18 and 29.41 respectively. The reduction in all the groups from baseline to four weeks, four weeks to eight weeks and from baseline to eight weeks is statistically significant.

Intra group comparison of plaque and bleeding indices for Groups A, B, C, D and E between baseline and four weeks; four weeks and eight weeks; baseline and eight weeks were statistically significant. Inter group comparison of plaque and bleeding indices for Group A, B, C, D and E between baseline and four weeks; four weeks and eight weeks; baseline and eight weeks were statistically insignificant.

Within a group, decrease in mean PI, BI, percentage reduction of plaque and bleeding index was observed. Percentage of plaque reduction is highest in Group B [76\%] at baseline to eight weeks and lowest in Group D [56\%]. Intra group comparisons of mean PI, mean BI, Percentage reduction of plaque and bleeding index are presented in graphs 1-4. Intergroup comparisons of PI, BI Percentage reduction of plaque and bleeding index are presented in Table 1, Table 2.

\section{Discussion}

To provide home-based oral hygiene more effectively, many types of manual and powered toothbrushes have been developed. However, manual toothbrushes were still the most promising option. A good toothbrush is relatively inexpensive compared to most dental procedures. Choosing the best toothbrush begins with choosing the right bristles designs. But selection of bristle design generally depends on individual preference. 
Table 1. Inter Group Comparisons of Plaque Index

\begin{tabular}{|c|c|c|c|c|}
\hline GROUPS & PLAQUE INDEX & BASELINE-4WEEKS & BASELINE-8WEEKS & 4 WEEKS-8 WEEKS \\
\hline \multirow{2}{*}{ GROUP A } & $\mathrm{MEAN} \pm \mathrm{SD}$ difference & $0.84 \pm 0.04$ & $0.97 \pm 0.06$ & $0.13 \pm 0.10$ \\
\hline & $\%$ of reduction & 58.74 & 67.83 & 22.03 \\
\hline \multirow{2}{*}{ GROUP B } & $\mathrm{MEAN} \pm \mathrm{SD}$ difference & $0.70 \pm 0.42$ & $0.86 \pm 0.48$ & $0.16 \pm 0.06$ \\
\hline & $\%$ of reduction & 61.95 & 76.11 & 37.21 \\
\hline \multirow{2}{*}{ GROUP C } & $\mathrm{MEAN} \pm \mathrm{SD}$ difference & $0.81 \pm 0.04$ & $0.88 \pm 0.01$ & $0.07 \pm 0.03$ \\
\hline & $\%$ of reduction & 57.86 & 62.86 & 11.86 \\
\hline \multirow{2}{*}{ GROUP D } & $\mathrm{MEAN} \pm \mathrm{SD}$ difference & $0.90 \pm 0.11$ & $1.01 \pm 0.09$ & $0.11 \pm 0.02$ \\
\hline & $\%$ of reduction & 50.28 & 56.42 & 12.36 \\
\hline \multirow{2}{*}{ GROUP E } & $\mathrm{MEAN} \pm \mathrm{SD}$ difference & $0.82 \pm 0.10$ & $0.92 \pm 0.10$ & $0.10 \pm 0.00$ \\
\hline & $\%$ of reduction & 53.59 & 60.13 & 14.08 \\
\hline P VALUE & & 0.656 & 0.706 & 0.142 \\
\hline
\end{tabular}

Table 2. Inter Group Comparisons of Bleeding Index [*- Statistically Significant]

\begin{tabular}{|c|c|c|c|c|}
\hline GROUPS & BLEEDING INDEX & BASELINE-4WEEKS & BASELINE-8WEEKS & 4 WEEKS-8 WEEKS \\
\hline \multirow{2}{*}{ GROUP A } & MEAN \pm SD difference & $0.14 \pm 0.04$ & $0.22 \pm 0.06$ & $0.08 \pm 0.02$ \\
\hline & $\%$ of reduction & 29.17 & 45.83 & 23.53 \\
\hline \multirow{2}{*}{ GROUP B } & MEAN \pm SD difference & $0.13 \pm 0.08$ & $0.19 \pm 0.14$ & $0.06 \pm 0.06$ \\
\hline & $\%$ of reduction & 35.14 & 51.35 & 30.00 \\
\hline \multirow{2}{*}{ GROUP C } & MEAN \pm SD difference & $0.05 \pm 0.04$ & $0.07 \pm 0.05$ & $0.02 \pm 0.01$ \\
\hline & $\%$ of reduction & 21.74 & 30.43 & 11.11 \\
\hline \multirow{2}{*}{ GROUP D } & MEAN \pm SD difference & $0.11 \pm 0.03$ & $0.15 \pm 0.09$ & $0.04 \pm 0.06$ \\
\hline & $\%$ of reduction & 33.33 & 45.46 & 18.18 \\
\hline \multirow{2}{*}{ GROUP E } & MEAN \pm SD difference & $0.05 \pm 0.08$ & $0.10 \pm 0.03$ & $0.05 \pm 0.01$ \\
\hline & $\%$ of reduction & 22.73 & 45.45 & 29.41 \\
\hline P VALUE & & $0.004^{*}$ & $0.010^{*}$ & $0.004^{*}$ \\
\hline
\end{tabular}

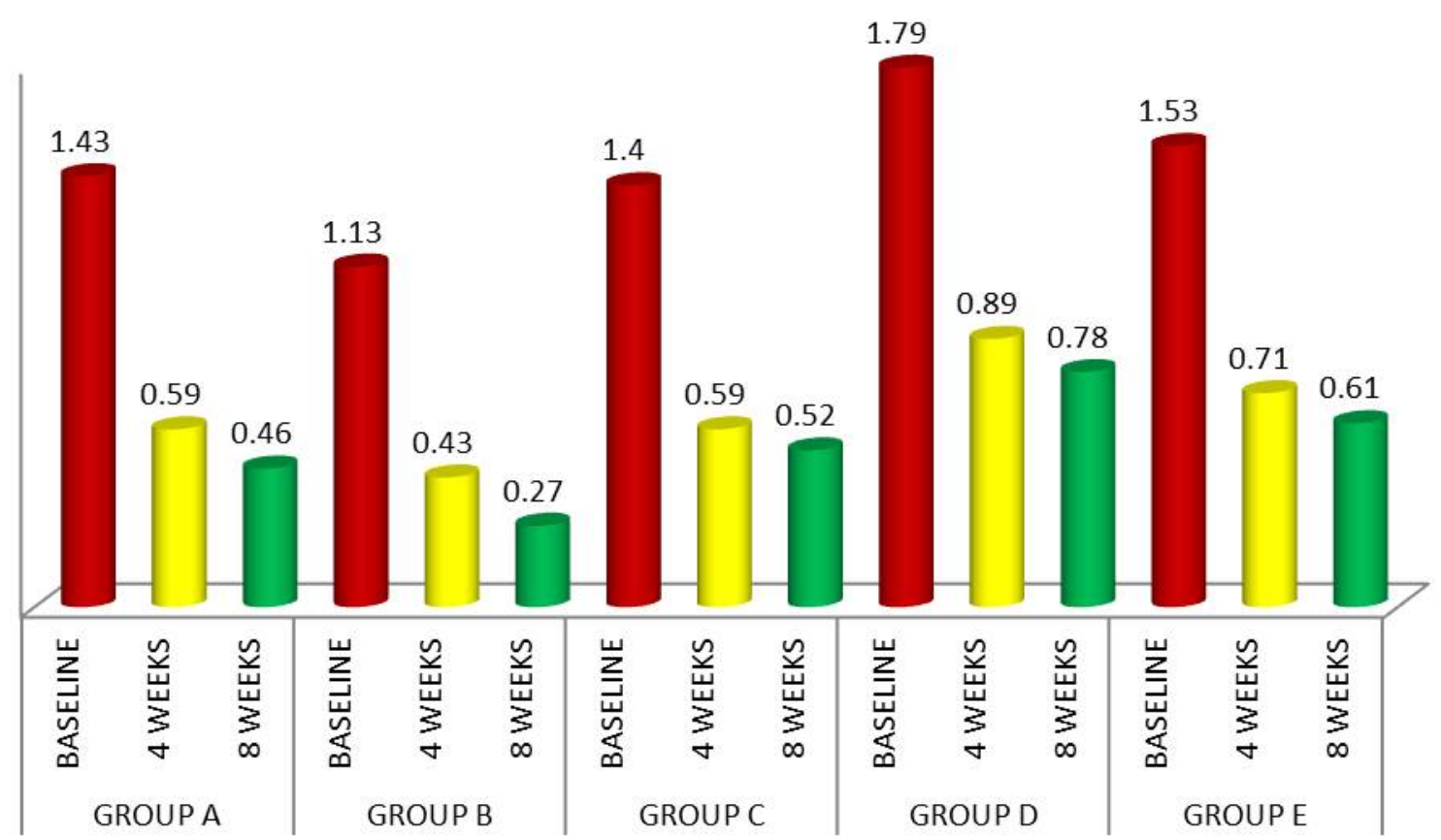

Graph 1. Mean Plaque Index for Individual Groups 


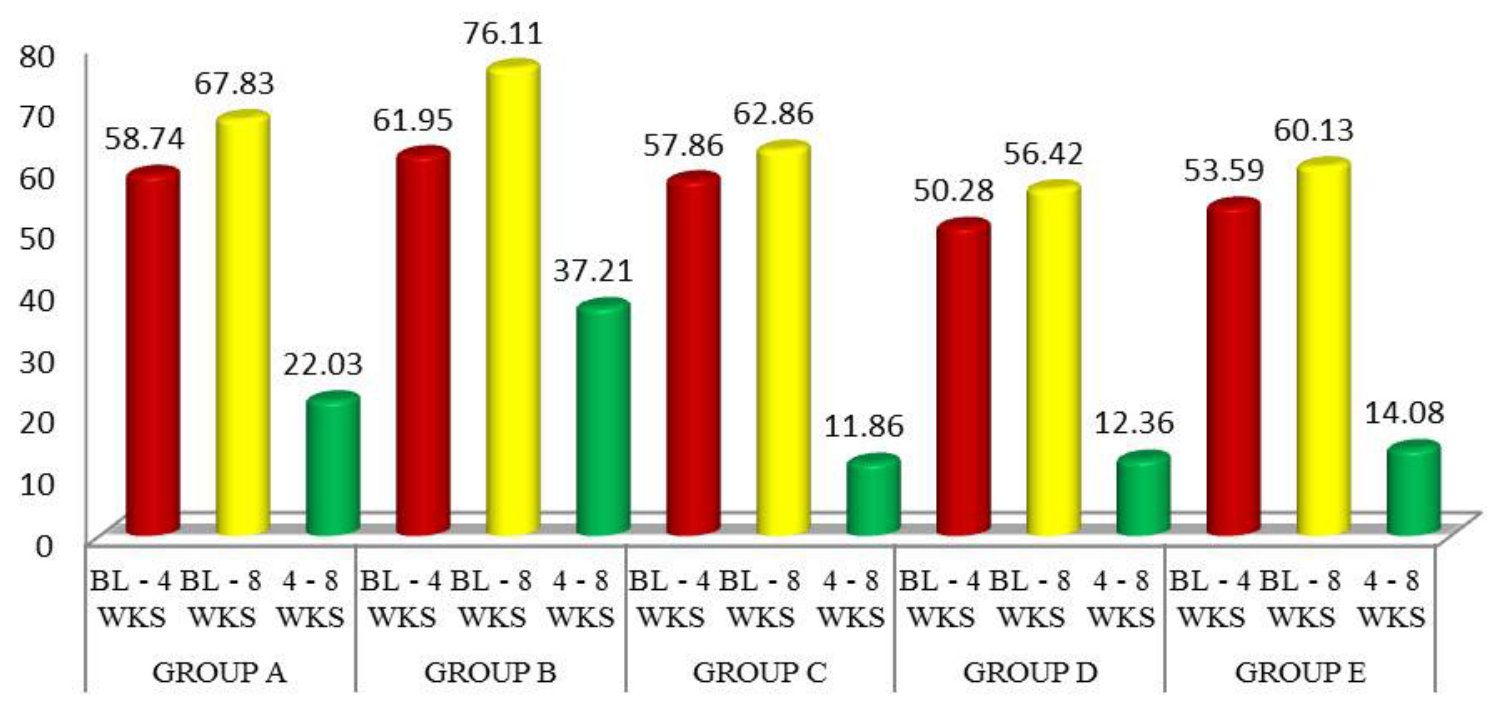

Graph 2. Percentage Plaque Reduction for Individual Groups

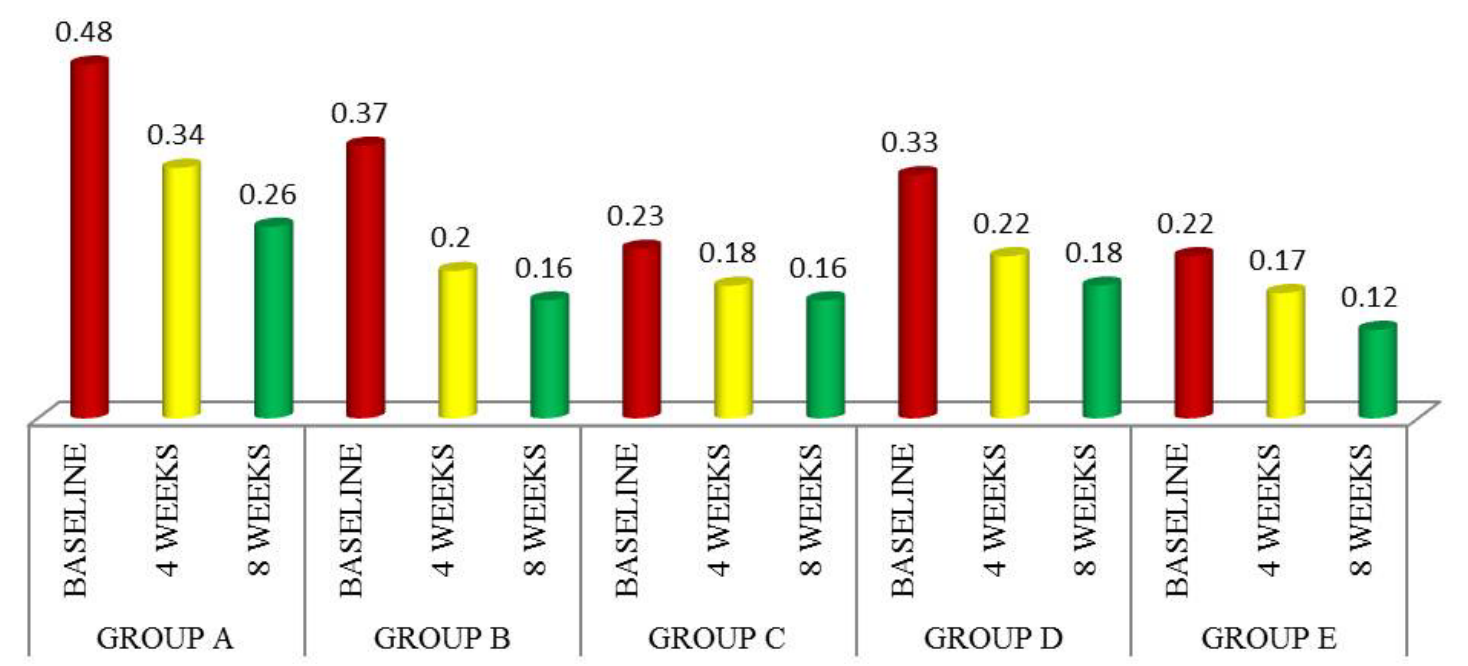

Graph 3. Mean Bleeding Index for Individual Groups

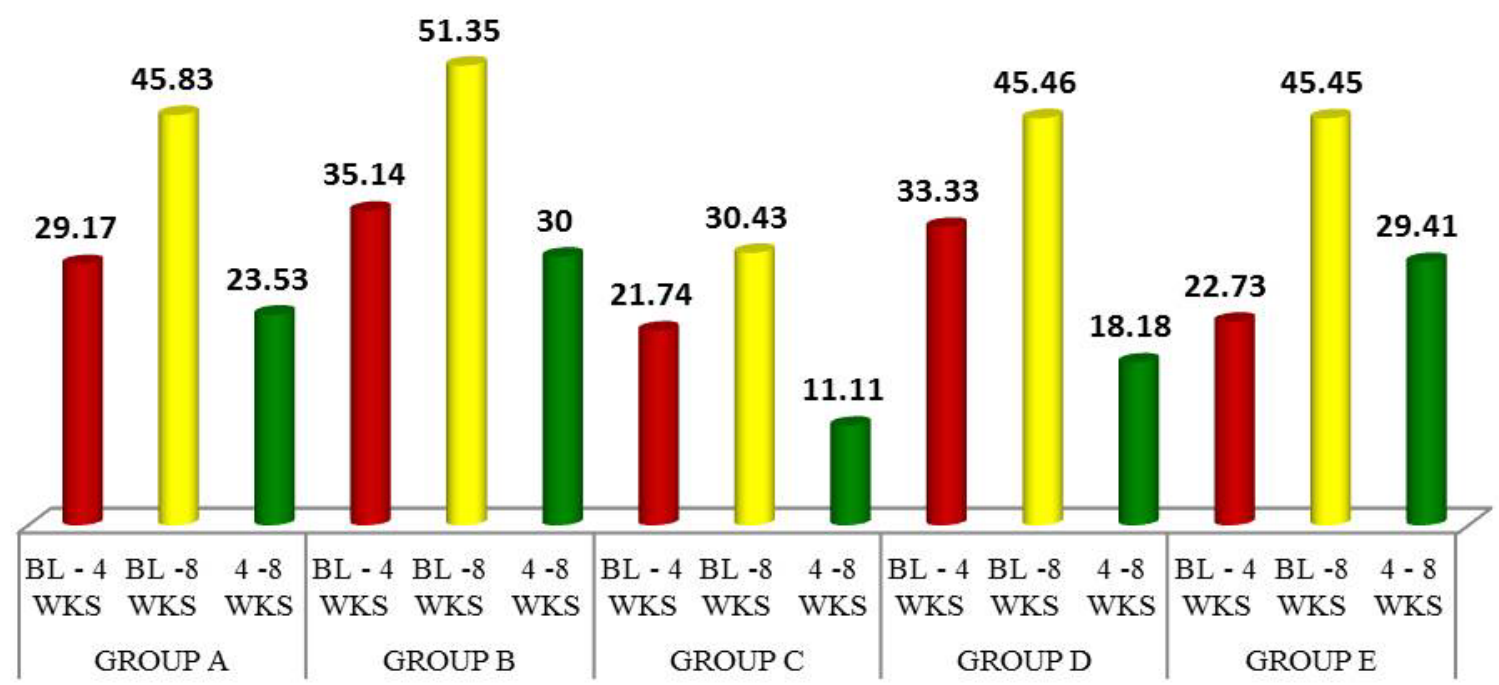

Graph 4. Percentage reduction in bleeding for Individual Groups

Although several workshops and reviews have consistently concluded that there is no superior design of manual toothbrush, yet different companies are coming out with different designs, each claiming superiority, backed by the results of their own clinical research team. So this study was put forth to evaluate the plaque removal efficacy of five commercially available toothbrushes.
Within a group, percentage of plaque and bleeding scores at base line, 4 weeks, and 8 weeks was reduced consistently. This emphasizes that tooth brushes are effective in reduction in percentage of plaque accumulation. Percentage of plaque reduction In Group A [Colgate Super shine] - 67.83\% and Group C [Oral B allrounder] - $62.86 \%$ is more than Group D [Oral B Fresh 
Clean] $-56.42 \%$. These observations are in accordance with Moustafa et al. [5] who compared a circular tooth brush with conventional tooth brush and found significant $(P<.05)$ improved plaque removal.

Percentage of plaque reduction is least [56.42\%] in Group D, [Oral B Fresh Clean] [Flat Rim ] This might be due to "blocking effect" of tight, dense bristle tufts which prevents individual tufts from entering the interproximal areas and once independent motion is achieved, the longer bristles can effectively reach farther between the teeth [6].

The results of the present study showed no statistically significant differences between five brushes which are in accordance with Bergenholtzet al. [7] who conducted a similar study comparing the toothbrush having v-shaped bristles with a flat-trim toothbrush and concluded no significant differences between the toothbrushes. This is also similar to Staudtet al. [8] who compared three toothbrushes namely convex bristle, multilevel bristle and flat trim bristles and was based on computer planimetric plaque index. The present study is in accordance with the literature that there is no superior design of manual toothbrush $[9,10,11]$.

The results of the present study are in contrast to Rustogiet al. [12], Sharma et al. [13], Singh et al. [14] who employed Navy index to quantify the plaque. This study does not hold in accordance with the manufacturer funded study conducted by Sharma et al. [15], who compared five toothbrushes having flat trimmed bristles, multi-level straight bristles with three advanced crisscross bristles and concluded that advanced crisscross bristles were superior among them. Even though statistically insignificant, Group B [Colgate Zigzag] has shown increase in percentage reduction of plaque index similar to Turner et al. [16] and Kakaret al [17].

Forward [18] stated that dentifrice improves brushing efficiency. Rustogiet al. [19] has reported retardation of plaque growth with a dentifrice. Keller and Manson-Hing [20] stated that a brush with dentifrice was more significantly more effective than was only a brush and water. So a common tooth paste is provided to the participants.

Single-use comparative clinical studies are useful for determining the relative plaque removal effectiveness of toothbrushes which may provide an indication of gingival health benefits long term. [21,22,23] Many indices have been developed and modified for clinical evaluation of the cleaning capacities of toothbrushes. According to Fischman, [24] the modified Turesky methods have been suggested as acceptable indices for the estimation of cleansing ability.

According to Gibson et al. [25] the mean plaque scores of all the teeth except third molars for each individual for each visit, were assessed. This provided more sensitive and accurate evaluation of brushing effectiveness compared with methods used in other studies like Bay et al. [26] and Scoppet al. [27] where only designated teeth were assessed. Bay et al. [26] utilized a standardized 3 minute brushing time. Shorter periods have been found to be inadequate.

However, further long term studies with large sample size are required for further assessment of the plaque removal efficacy.

\section{Conclusion}

Toothbrush continues to be the most promising plaque control device. Of the many factors which influence plaque removal ability, bristle design has been widely studied. The present study showed no significant differences between the five toothbrushes in plaque removal efficacy. This supports to the previous studies that there is no superior manual tooth brush. This may lead to a conclusion that the individual skills are more concerned in plaque removal efficacy.

\section{References}

[1] Loe H, Theilade E, Jensen SB. Experimental gingivitis in man. $J$ Periodontol1965; 36: 177-87.

[2] Van der Weijden FA, Campbell SL, Dorfer CEet al. Safety of oscillating-rotating powered brushes compared to manual toothbrushes: a systematic review. J Periodontol2011; 82: 5-24.

[3] Turesky S, Gilmore ND, Glickman I. Reduced plaque formation by the chloromethyl analogue of victamine C.J Periodontol 1970; 41: 41-3.

[4] Mombelli A, Van Oosten MA, Schurch E Jr et al. The microbiota associated with successful or failing osseointegrated titanium implants. Oral MicrobiolImmunol 1987; 2: 145-51.

[5] Moustafa MH, Shoeib MA, el-Din MS et al. Clinical evaluation of the efficacy of two different designs of toothbrushes in plaque removal. Egypt Dent J I986; 12: 131-50.

[6] Mintel TE, Crawford J. The search for a superior toothbrush design technology. J Clin Dent 1992; 3: C1-C4.

[7] Bergenholtz A, Gustafsson LB, Segerlund $\mathrm{N}$ et al. Role of brushing technique and toothbrush design in plaque removal. Scand J Dent Res 1984; 92: 344-51.

[8] Staudt CB, Kinzel S, Hassfeld S et al. Computer based intra-oral image analysis of the clinical plaque removing capacity of 3 manual toothbrushes. J ClinPeriodontol 2001; 28: 746-52.

[9] Frandsen A. Mechanical oral hygiene practices. In: Loe $\mathrm{H}$, Kleinman M, editors. Dental plaque control measures and oral hygiene practices. 1st ed. IRL Press: Oxford; 1986. p. 93-116.

[10] Jepsen S. Role of manual toothbrushes in effective plaque control: Advantages and limitations. In: Lang NP, Attstrom R, Love H, editors. Proceedings of European workshop on Mechanical plaque control. 1st ed. Quintessence Publishing: Illinois; 1983. p. 121-37.

[11] Addy M. Measuring success in toothbrush design- An opinion and debate of concepts. Int Dent J 1998; 48: 509-18.

[12] Rustogi KN, Curtis JP, Volpe AR et al. Refinement of the Modified Navy Plaque Index to increase plaque scoring efficiency at the gum line and interproximal tooth areas. J Clin Dent 1992; 3: C9-12.

[13] Sharma NC, Galustians J, Rustogi KN et al.Comparative plaque removal efficacy of three toothbrushes in two independent clinical studies. J Clin Dent 1992; 3: C13-20.

[14] Singh SM, Rustogi KN, McCool, JJ et al. Clinical studies regarding the plaque removal efficacy of manual toothbrushes. $J$ Clin Dent 1992; 3: C21-8.

[15] Sharma NC, Qaqish J, Walters PAet al. A clinical evaluation of the plaque removal efficacy of five manual toothbrushes. $J$ Clin Dent 2010; 21: 8-12.

[16] Turner PS, Surveyor AB, Turner FP. A clinical comparison of plaque removal of five manual toothbrushes. J Indian Dent Assoc 1999; 70: 77-81.

[17] Kakar A, Kakar RC, Kakar K,et al. Plaque removing efficacy of a new design toothbrush with Zig-Zag bristle arrangement. J Indian Dent Assoc2002; 73: 29-34.

[18] Forward GC. Role of toothpastes in the cleaning of teeth. Int Dent $J$ 1991:41:164-70.

[19] Rustogi KN, Volpe AR, Fishman Set al. Removal of 48-hour plaque by either brushing with dentifrices or water. $J$ Dent Res 1984, 63: 312.

[20] Keller SE, Manson-Hing LR. Clearance studies of proximal tooth surfaces. II: in vivo removal of interproximal plaque. Ala J Med Sci 1969; 6: 266-74.

[21] Claydon N, Addy M. Comparison single-use plaque removal by toothbrushes of different designs. J ClinPeriodontol. 1996; 23: $1112-6$. 
[22] Sharma NC, Goyal CR, Qaqish JG et al. Single-use plaque removal efficacy of three power toothbrushes. J Dent 2005; 33S1: $11-5$.

[23] Sharma NC, Qaqish JG, Galustians HJ et al. Plaque removal efficacy of two electric toothbrushes with different brush head designs. J Dent 2005; 33Suppl 1:17-21.

[24] Fischman, SL. Current status of indices of plaque. $J$ ClinPeriodontol. 1986; 13: 371-4.
[25] Gibson MT, Joyston-Bechal S, Smales FC. Clinical evaluation of plaque removal with double headed toothbrush. J ClinPeriodontol. 1988: 15: 94-8.

[26] Bay I, Kardel KK, Skouggard MR. Quantitative evaluation of the plaque-removing ability of different types of toothbrushes. $J$ Periodontol. 1967; 38: 526-33.

[27] Scopp IW, Cohen G, Cancro LP et al. Clinical evaluation of a newly designed contoured toothbrush. J Periodontol 1976; 47: 8790 . 